\title{
Caracterização e Evolução dos Internamentos Evitáveis em Portugal: Impacto de Duas Abordagens Metodológicas
}

\author{
Characterization and Evolution of Avoidable Admissions in Portugal: The \\ Impact of Two Methodologic Approaches
}

\author{
João SARMENTO ${ }^{1}$, Conceição ALVES ${ }^{2}$, Paula OLIVEIRA ${ }^{2}$, Rita SEBASTIÃO ${ }^{3}$, Rui SANTANA ${ }^{4}$ \\ Acta Med Port 2015 Sep-Oct;28(5):590-600
}

\section{RESUMO}

Introdução: O objectivo deste estudo é avaliar a performance dos sistemas de saúde através dos internamentos evitáveis, dado que estes têm vindo a ganhar importância a nível internacional. Foram utilizadas duas metodologias distintas para a identificação dos internamentos por Ambulatory Care Sensitive Conditions, fazendo uma descrição da realidade e a evolução destes em Portugal.

Material e Métodos: Analisámos mais de 12 milhões de internamentos entre 2000 e 2012, utilizando as bases de dados de resumos de alta nacionais. Utilizaram-se duas metodologias de identificação das Ambulatory Care Sensitive Conditions, determinando a sua concordância. Calculámos cenários de melhoria potencial.

Resultados: Em 2012, 4,4\% e 32,5\% dos internamentos por causas médicas seriam evitáveis segundo a metodologia canadiana e espanhola respectivamente. Os internamentos são mais frequentes nas crianças e nos idosos, divergindo as suas causas de acordo com o grupo etário e a metodologia utilizada. A taxa de internamentos no período em análise diminuiu $20 \%$ segundo a metodologia canadiana e aumentou $16 \%$ segundo a espanhola. Existem agregados regionais de performance acima e abaixo da média nacional. A concordância entre as duas metodologias é baixa. Estimaram-se reduções potenciais entre $20,3 \%$ e $53,5 \%$ dos internamentos.

Discussão: Os internamentos evitáveis assumem um volume considerável em Portugal. Apesar de em teoria serem evitáveis a sua eliminação por completo não é uma possibilidade prática, no entanto, o seu estudo possibilita a avaliação e monitorização de resultados e o estabelecimento de prioridades de intervenção

Conclusão: Para uma caracterização mais precisa dos internamentos evitáveis é necessário consensualizar em Portugal a metodologia para a sua identificação.

Palavras-chave: Avaliação de Processos e Resultados (Cuidados de Saúde); Cuidados Ambulatórios; Hospitalização; Portugal.

\section{ABSTRACT}

Introduction: The aim of this study is to evaluate the health systems performance through the avoidable hospital admissions, once these have gained international relevance. We used two different methods to identify the admissions for Ambulatory Care Sensitive Conditions, describing the Portuguese reality and evolution.

Material and Methods: Over 12 million hospitalizations were analyzed between 2000 and 2012 using the national hospital discharge databases. We used two different methodologies to identify the hospitalizations for Ambulatory Care Sensitive Conditions, determining their concordance. We also estimated potential improvement scenarios.

Results: In $2012,4.4 \%$ and $32.4 \%$ of the hospitalizations for medical causes were avoidable according to the Canadian and Spanish methodologies respectively. The hospitalizations are more frequent in children and the elderly. The most frequent causes vary according to the age group and methodology. During the analyzed period the rate of admissions has dropped $20 \%$ according to the Canadian methodology and increased $16 \%$ according to the Spanish methodology. There are regional clusters of performance under and above the national average. The concordance between methodologies is low. The improvement scenarios estimated possible reductions between $20.3 \%$ and $53.5 \%$ of the hospitalizations.

Discussion: The avoidable admissions assume a relevant volume in Portugal. Although in theory they are avoidable their complete elimination is a practical impossibility. Their study, however, allows the evaluation and results motorization enabling to establish intervention priorities.

Conclusion: To have a precise characterization of the avoidable admissions in Portugal it is necessary to achieve consensus on the identification methodology.

Keywords: Ambulatory Care; Hospitalization Outcome and Process Assessment (Health Care); Portugal.

\section{INTRODUÇÃO}

A utilização dos internamentos evitáveis como indicador de performance dos sistemas de Saúde tem vindo a ganhar importância a nível internacional, como demonstram diversos estudos realizados no Canadá, Reino Unido, Austrália e Espanha. ${ }^{1-4}$ No Reino Unido, país com um sistema nacional de Saúde semelhante ao português em muitas dimensões, um em cada seis episódios de urgência são por causas evi- táveis, com um impacto financeiro no serviço nacional de saúde Inglês de cerca de $£ 1,42$ mil milhões. ${ }^{2}$ A ocorrência de um internamento evitável geralmente significa que se perdeu uma oportunidade de intervir preventivamente no percurso de cuidados do utente. Pode também significar que os cuidados prestados em ambulatório não estão a ser efectivos. ${ }^{5,6}$

1. Escola Nacional de Saúde Pública (ENSP). Universidade NOVA de Lisboa. Lisboa. Portugal.

2. Unidade de Saúde Familiar São Julião. Oeiras. Portugal.

3. Unidade de Cuidados de Saúde Personalizados de Sete Rios. Lisboa. Portugal.

4. Centro de Investigação em Saúde Pública (CISP). Escola Nacional de Saúde Pública (ENSP). Universidade NOVA de Lisboa. Lisboa. Portugal.

$\bowtie$ Autor correspondente: João Sarmento. j.sarmento@ensp.unl.pt

Recebido: 16 de Fevereiro de 2015 - Aceite: 01 de Setembro de 2015 | Copyright @ Ordem dos Médicos 2015 
Uma das formas possíveis de medição dos internamentos evitáveis é através da análise das Ambulatory Care Sensitive Conditions (ACSC). As ACSC, no seu conceito mais lato, são um conjunto de patologias para as quais existe conhecimento e tecnologia para a sua prevenção e/ ou tratamento sem recurso ao internamento hospitalar. ${ }^{6,7}$ Assim, a falta de acesso a cuidados de ambulatório, ou a sua falta de efectividade podem resultar em internamentos evitáveis. ${ }^{6}$ Existem três dimensões de actividades que promovem a evicção de internamentos: a) prevenção primária (p.ex. vacinação); b) diagnóstico e tratamento precoce/ atempado e c) bom controlo e gestão da doença crónica. ${ }^{8}$ Todas estas actividades encontram-se primordialmente atribuídas aos Cuidados de Saúde Primários (CSP). De facto, é geralmente aceite na literatura internacional que as ACSC devem ser utilizadas como indicador de acesso e efectividade dos CSP. ${ }^{6,8-10}$ Por esta razão, a utilização da tradução literal 'Internamentos por causas sensíveis a cuidados de ambulatório', pode ter, em Portugal, uma interpretação dúbia. É possível incluir na designação 'cuidados de ambulatório' actividades tão díspares como os CSP, hospital de dia, consulta externa ou cirurgia de ambulatório. Apesar destas actividades também contribuírem para a evicção de internamentos, a utilização das ACSC está validada para a avaliação da performance dos CSP, a um nível sistémico, não havendo evidência para a sua utilização a nível micro (p.ex. avaliação individual de prestadores ou equipas de prestadores). ${ }^{6,8}$ Noutro país lusófono a tradução utilizada é 'Internações por causas sensíveis à atenção primária', ${ }^{10}$ clarificando a atribuição do efeito de evicção dos internamentos especificamente aos CSP. Pelas razões expostas, consideramos conceptualmente mais adequada a denominação 'Internamentos por causas sensíveis aos Cuidados de Saúde Primários' (ICSCSP).

Um dos principais desafios na análise desta problemática é a definição das próprias ACSC. Actualmente existe uma discussão alargada em todo o mundo, principalmente no Canadá, Estados Unidos da América, Austrália, Reino Unido, Espanha e Brasil acerca da definição e utilização deste indicador na avaliação dos Sistemas de Saúde. A análise conceptual é determinante. Como discutido anteriormente, ao conceito destes internamentos está inerente uma acção dos CSP, pelo que para se considerar um internamento evitável devem estar reunidas as seguintes condições ${ }^{6}$ :

a) Existir tecnologia/conhecimento para prevenir/tratar a patologia em questão sem recurso ao internamento hospitalar, de forma efectiva e segura para a pessoa;

b) A acção necessária para a evicção do internamento depende dos prestadores de CSP.

Deve existir consenso sobre quais os internamentos evitáveis em relação ao contexto específico de cada Sistema de Saúde..$^{4,6}$ A determinação da metodologia de identificação dos internamentos evitáveis deve ter uma clara fundamentação técnica, incorporando as características específicas do sistema de saúde em que será aplicada, sendo clara qual a perspectiva e escopo da análise pretendida. É recomendável a utilização de critérios específicos que garantam a selecção de problemas de saúde relevantes e em relação aos quais a acção dos prestadores seja determinante. ${ }^{11-13}$ Salientam-se os critérios utilizados por Caminal et al ${ }^{11}$ para a selecção de ACSC na Catalunha, pela sua abrangência e especificidade:

- Critério a) evidência de utilização prévia na literatura;

- Critério b) taxa de internamentos relevante (> 1/10 000 hab.);

- Critério c) clareza na definição e codificação do diagnóstico;

- Critério d) responsabilidade dos CSP na acção para evicção do internamento;

- Critério e) necessidade de hospitalização em caso de diagnóstico.

Os ICSCSP são influenciados por vários fatores relacionados com o contexto onde são medidos, sendo ampla a evidência neste campo. Sistematicamente é possível organizar estes fatores em quatro grandes grupos de características:

a) Ligadas aos prestadores;

b) Socioeconómicas e demográficas;

c) Geográficas;

d) Epidemiológicas.

A estrutura e processos dos prestadores de cuidados de saúde influenciam os ICSCSP, quer ao nível dos CSP, hospitais e cuidados continuados, quer na relação entre estes níveis de cuidados. Ao nível dos CSP existe uma relação inversa entre o número de ICSCSP e o número de médicos de família por 100000 habitantes, ${ }^{3,14}$ bem como o número de consultas com o médico de família.,14,15 Outro aspecto fundamental é a continuidade de cuidados praticada nos CSP. Verificou-se que os utentes com mais de $75 \%$ das suas consultas com o mesmo médico de família têm uma menor probabilidade de serem internados. ${ }^{16}$

As características socioeconómicas e demográficas da população têm influência nos ICSCSP. Estes são mais frequentes nos elementos do sexo masculino, com dois picos de incidência, nomeadamente nas faixas etárias superiores aos 65 anos e inferiores aos 15 anos. ${ }^{11,14} \mathrm{~A}$ associação entre o número de ICSCSP e os indicadores socioeconómicos tem sido alvo de vários estudos por todo o mundo. ${ }^{3,17-20}$ Todos eles têm evidenciado uma relação inversa. Da mesma forma, níveis mais baixos de escolaridade e níveis mais elevados de desemprego parecem relacionar-se com taxas de ICSCSP mais elevadas..$^{5,8}$

As características geográficas onde se inserem os prestadores também influenciam os ICSCSP. A distância ao hospital parece influenciar os internamentos, nomeadamente, aumentando o seu número quanto mais próximo se estiver deste. ${ }^{11,21}$ Por outro lado, segundo Ansari, ${ }^{3}$ a menor densidade populacional e o maior isolamento parecem ser preditores de maiores taxas de ICSCSP.

Finalmente, a incidência, prevalência e severidade das patologias em determinada área geográfica estão inegavelmente relacionadas com as taxas de ICSCSP. ${ }^{3}$ 
Tabela 1 - Comparação entre as metodologias de identificação dos ICSCSP segundo Caminal et al (metodologia espanhola) e CIHI (metodologia canadiana)

\begin{tabular}{|c|c|c|}
\hline \multirow[b]{2}{*}{ Patologia } & \multicolumn{2}{|c|}{$\begin{array}{l}\text { Códigos ICD-9CM } \\
\text { (Diagnóstico Principal) }\end{array}$} \\
\hline & Metodologia espanhola & Metodologia canadiana \\
\hline \multicolumn{3}{|l|}{ I - Doenças infecciosas evitáveis por vacinação ou outros: } \\
\hline Difteria & 032 & \\
\hline Tétano & 037 & \\
\hline Poliomielite & 045 & \\
\hline Meningite por hemófilos & 320.0 & \\
\hline Febre reumática & 390,391 & \\
\hline \multicolumn{3}{|l|}{ II - Sífilis congénita } \\
\hline Sífilis congénita & 090 & \\
\hline \multicolumn{3}{|l|}{ III - Tuberculose } \\
\hline Outras tuberculoses & $012-018$ & \\
\hline \multicolumn{3}{|l|}{ IV - Infecções respiratórias superiores } \\
\hline Abcesso periamigdalino & 475 & \\
\hline \multicolumn{3}{|l|}{ V-Pneumonia } \\
\hline Pneumonia & $482.2,482.3,483,485,486$ & \\
\hline \multicolumn{3}{|l|}{$\mathrm{VI}$ - Infecções do trato urinário } \\
\hline Pielonefrite aguda & 590.1 & \\
\hline \multicolumn{3}{|l|}{ VII - Apendicite perfurada } \\
\hline Apendicite aguda com peritonite generalizada & 540.0 & \\
\hline Apendicite aguda com abcesso peritoneal & 540.1 & \\
\hline \multicolumn{3}{|l|}{ VIII - Úlcera sangrante ou perfurada } \\
\hline $\begin{array}{l}\text { Úlcera gástrica aguda ou crónica ou n. esp., c/ hemorragia } \\
\text { ou c/ hemorragia e perfuração }\end{array}$ & $531.0,531.2,531.4,531.6$ & \\
\hline $\begin{array}{l}\text { Úlcera duodenal aguda ou crónica ou n. esp., c/ hemorragia } \\
\text { ou c/ hemorragia e perfuração }\end{array}$ & $532.0,532.2,532.4,532.6$ & \\
\hline $\begin{array}{l}\text { Úlcera péptica, sitio n. esp., aguda ou crónica ou n. esp., c/ } \\
\text { hemorragia ou c/ hemorragia e perfuração }\end{array}$ & $533.0,533.2,533.4,533.6$ & \\
\hline \multicolumn{3}{|l|}{ IX - Distúrbios hidroeletrolíticos } \\
\hline Desidratação & 276.5 & \\
\hline Hipocaliemia & 276.8 & \\
\hline \multicolumn{3}{|l|}{ X-Epilepsia } \\
\hline Epilepsia e grande mal convulsivo & & 345 \\
\hline \multicolumn{3}{|l|}{$\mathrm{XI}$ - Asma } \\
\hline Asma & \multicolumn{2}{|c|}{493} \\
\hline \multicolumn{3}{|l|}{ XII - Doença pulmonar obstrutiva crónica (DPOC) } \\
\hline DPOC & \multicolumn{2}{|c|}{ (apenas se existe um diagnóstico secundário 496) } \\
\hline \multicolumn{3}{|l|}{ XIII - Diabetes } \\
\hline Diabetes & $250.1,250.2,250.3$ & $250.0,250.1,250.2,250.8$ \\
\hline Coma hipoglicémico & 251.0 & \\
\hline Gangrena + diabetes & $\begin{array}{l}785.4 \text { (apenas se existe um } \\
\text { diagnóstico secundário } 250.7 \text { ) }\end{array}$ & \\
\hline
\end{tabular}

$\mathrm{Na}$ literatura, estão disponíveis várias metodologias de identificação de ICSCSP criadas para diferentes realidades. ${ }^{8,9,11,13,22-26}$ Este facto cria uma dificuldade de com- paração internacional dos resultados, no entanto, ganha-se especificidade pela maior adequação da metodologia às responsabilidades e ações dos CSP de cada país. 
Tabela 1 - Comparação entre as metodologias de identificação dos ICSCSP segundo Caminal et al (metodologia espanhola) e CIHI (metodologia canadiana) (continuação)

Códigos ICD-9CM

(Diagnóstico Principal)

Patologia

Metodologia espanhola

Metodologia canadiana

XIV - Patologia cardiovascular e hipertensão arterial

Hipertensão essencial maligna

401.0

$401.0^{*}$

Hipertensão essencial não especificada como maligna ou benigna

Doença cardíaca hipertensiva

Doença renal hipertensiva

Doença cardíaca e renal hipertensiva

Hipertensão secundária maligna

405.0

Doença cardíaca isquémica

$410-414$

Doença cerebrovascular

$430,431,436,437.2$

XV - Insuficiência cardíaca (IC)

Doença cardíaca hipertensiva maligna com IC

402.01

Doença cardíaca hipertensiva benigna com IC

402.11

Doença cardíaca hipertensiva não especificada c/ IC

Insuficiência cardíaca

402.91

428

$428 *$

Edema agudo do pulmão não especificado

518.4

$518.4^{*}$

XVI - Angina

Síndrome coronário intermediário

$411.1^{*}$

Forma aguda ou subaguda de doença isquémica do

coração, ncop

Angina de peito

$411.8^{*}$

$413^{*}$

XVII - Doença inflamatória pélvica

Doença inflamatória do ovário, trompa de Falópio, tecido celular pélvico e peritoneu

614

${ }^{*}$ Códigos de procedimentos cardíacos para exclusão: ICD-9-CM: $336,35^{\wedge}, 36^{\wedge \wedge}, 373^{\wedge}, 375^{\wedge}, 377^{\wedge}, 378^{\wedge}$, 3794-3798. Critérios de exclusão da metodologia Canadiana: a) Morte antes da alta; b) Idade superior a 75 anos.

Em Portugal a utilização das ACSC tem sido aflorada, havendo uma indefinição clara quanto à metodologia de identificação. O Alto Comissariado da Saúde sugeriu a incorporação deste conceito como indicador de ganhos em Saúde no Plano Nacional de Saúde 2011-2016 ${ }^{27}$ (documento estratégico em vigor, tendo sido aprovada em Maio a sua extensão até 2020), utilizando a metodologia desenvolvida pelo Canadian Institute for Health Information $(\mathrm{ClHI}) .{ }^{26} \mathrm{Um}$ trabalho académico com o objectivo de avaliar o acesso aos $\mathrm{CSP}^{28}$ utilizou a metodologia de Billings et $a^{23} \mathrm{e}$ finalmente uma análise de uma consultora da área da Saúde ${ }^{29}$ utilizou uma adaptação parcial da metodologia de Caminal et al. ${ }^{11}$ Para exemplificar a disparidade de resultados obtidos pela utilização de diferentes metodologias (analisado em maior detalhe adiante) o custo financeiro estimado para o SNS seria de $45610708 €$ e $450234825 €$ de acordo com as metodologias do $\mathrm{ClHI}$ e Caminal et al, respectivamente. Não é do conhecimento dos autores qualquer trabalho de validação de uma lista de ACSC adaptada à realidade portuguesa, que urge face ao exposto.

\section{Objectivos}

- Determinar e caracterizar os ICSCSP em Portugal;

- Caracterizar a evolução histórica da taxa de ICSCSP;

- Determinar potenciais cenários de melhoria;

- Avaliar o impacto da utilização de diferentes metodologias de identificação dos ICSCSP em Portugal.

\section{MATERIAL E MÉTODOS}

Realizou-se um estudo observacional, retrospectivo, descritivo utilizando os dados relativos a 12467427 internamentos de cidadãos residentes no território continental português, entre o ano de 2000 e 2012. Excluímos os episódios de ambulatório (p. ex. cirurgia de ambulatório e hospital de dia). Os dados foram cedidos pela Administração Central dos Serviços de Saúde (ACSS), sendo provenientes das bases de dados de resumos de altas dos hospitais públicos do território continental português, após anonimização.

Determinámos os ICSCSP com base no código de diagnóstico principal, segundo a International Classification of $D i-$ seases Nineth Revision Clinical Modification (ICD-9CM), de acordo com duas metodologias internacionais: a do $\mathrm{CIHI}^{27} \mathrm{e}$ a validada por Caminal et al ${ }^{11}$ em Espanha (Tabela 1). 
Calculámos as taxas de ICSCSP $\left(T_{i n t}\right)$ com os dados do Instituto Nacional de Estatística referentes às estimativas populacionais anuais, por concelho $\left(\mathrm{Pop}_{i}\right){ }_{i}=$ concelho $)$. $\mathrm{T}_{\text {int }}=n \mathrm{ICSCSP}_{i} / \mathrm{Pop}_{i}$

As taxas de internamento foram padronizadas segundo o método directo, recorrendo à população padrão mundial da Organização Mundial de Saúde. ${ }^{30}$

Para calcular cenários de melhoria, os concelhos foram distribuídos em cinco quintis equidistantes com base na taxa padronizada de ICSCSP. Calcularam-se dois cenários de melhoria. ${ }^{2} \mathrm{O}$ cenário up the ladder pressupõe que é possível que em cada concelho haja uma melhoria até ao nível médio (de taxa de ICSCP) do quintil imediatamente melhor. O cenário all the best pressupõe que é possível que em todos os concelhos se atinga a taxa de ICSCSP média do melhor percentil.

Calculámos da estatística kappa como medida de concordância usada em escalas nominais, para avaliar o grau de concordância da classificação em percentis entre as duas metodologias de identificação dos ICSCSP. Os valores da estatística kappa variam entre 0 e 1 , sendo que ' 0 ' indica a não existência de concordância além do puro acaso, e ' 1 ' concordância perfeita. ${ }^{31}$

Por sua vez, a representatividade financeira (RF) dos ICSCSP foi estimada segundo a seguinte fórmula:

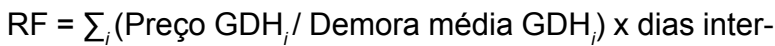
namento observados em que:

$\mathrm{RF}=$ representatividade financeira, valorizada em euros

$=I C S C S P$

Preço GDH = preço atribuído por grupo diagnóstico homogéneo (GDH), explícito na portaria 839-A/2009. ${ }^{32}$

Demora média GDH = demora média esperada por internamento explícita na portaria 839-A/2009. ${ }^{32}$

Dias de internamento observados $=$ número de dias de internamento reais em cada episódio.

Para estimar os ganhos financeiros potenciais nos cenários de melhoria definidos, utilizou-se o valor médio dos ICSCSP apurado segundo esta metodologia.

\section{RESULTADOS \\ ICSCSP em Portugal no ano de 2012}

Caracterização: Em 2012, os ICSCSP representaram $4,4 \%$ e $32,5 \%$ dos internamentos por causas médicas, segundo a metodologia canadiana e espanhola respectivamente. Os internamentos foram mais frequentes no sexo masculino: $57,0 \%$ (metodologia canadiana) e $52,0 \%$ (metodologia espanhola). A distribuição dos ICSCSP pelas classes etárias revela o padrão descrito na literatura. Segundo a metodologia canadiana, existiram dois picos de maior frequência (Fig. 1): o primeiro em idade pediátrica, essencialmente devido a internamentos por asma, e o segundo a partir da quinta década de vida, devido a

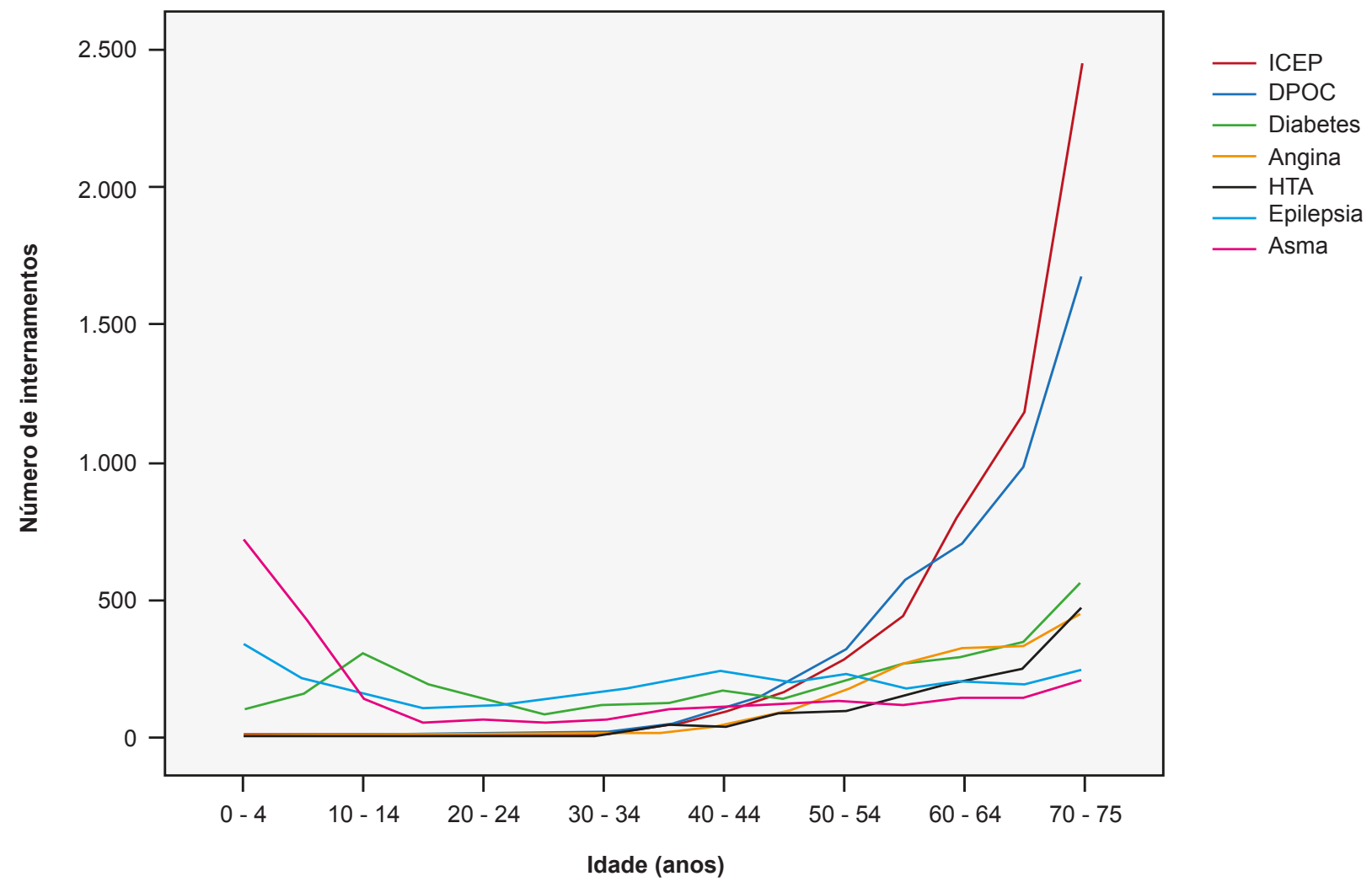

Figura 1 - Distribuição dos ICSCSP por patologia e classe etária segundo a metodologia canadiana

São excluídos internamentos de indivíduos com: a) Morte antes da alta; b) Idade superior a 75 anos; ICEP: Insuficiência cardíaca e edema agudo do pulmão; DPOC: Doença pulmonar obstrutiva crónica; HTA: Hipertensão arterial 
insuficiência cardíaca e DPOC. Segundo a metodologia espanhola, mantém-se o padrão (Fig. 2). No entanto, salienta-se um volumoso pico nos idosos (80-84 anos) referente a internamentos por pneumonia, insuficiência cardíaca e DPOC. Segundo esta metodologia, é também mais precoce o pico de internamentos por cardiopatia hipertensiva, a partir dos 45 - 49 anos. Em idade pediátrica, as causas mais frequentes de internamento diferem relativamente à metodologia canadiana. São mais relevantes os internamentos por pneumonia, pielonefrite e gastroenterite. As patologias que mais frequentemente motivaram internamentos foram diferentes consoante a metodologia utilizada. Segundo a canadiana, $20,9 \%$ dos ICSCSP deveram-se a DPOC, seguindo-se a insuficiência cardíaca e edema agudo do pulmão $(20,5 \%)$ e a diabetes $(15,1 \%)$. Segundo a metodologia espanhola, a pneumonia $(23,8 \%)$ e a cardiopatia hipertensiva $(20,6 \%)$ antecedem a insuficiência cardíaca $(12,6 \%)$ e a DPOC $(12,2 \%)$ como causas mais frequentes de internamento.

Distribuição geográfica: $\mathrm{A}$ análise do número total de internamentos não tem em conta as diferentes características das populações de cada concelho, quer em número de habitantes, quer em distribuição etária. Por essa razão, o estudo das taxas de internamento padronizadas permite um maior detalhe na análise geográfica do fenómeno dos ICSCSP ao eliminar as diferenças atribuíveis às características da população. Para facilitar a análise foram calculados cinco quintis equidistantes relativamente à taxa padronizada de internamentos. $O$ quintil 1 é referente às taxas mais baixas (Fig. 3). Utilizando a metodologia canadiana, as taxas (padronizadas) de internamento variam entre 24,1 e 517,8 int. $/ 100000$ hab. $(x=185,4 d p=72,6)$. É perceptível que existem aglomerados regionais de performance acima e abaixo da média. Pela positiva destacam-se os distritos de Évora, Beja, Porto e Braga, bem como a região do Algarve com resultados inferiores à média nacional. Pela negativa destacam-se os distritos de Portalegre, Castelo Branco, Leira e a Região de Trás-os-Montes e Alto Douro. Segundo a metodologia espanhola, os valores da taxa de internamento variaram entre 421,0 e 1805,5 int./100 000 hab. $(x=999,4 d p=220,8)$. O panorama é ligeiramente diferente, apesar de algumas tendências se manterem, nomeadamente os resultados acima da média em alguns concelhos do Alentejo, Algarve, Porto e Braga, bem como a tendência para resultados abaixo da média nacional nas regiões de Trás-os-Montes e Alto Douro, Beira Baixa e Centro Litoral.

Evolução dos ICSCSP em Portugal entre 2000 e 2012: Os resultados diferem de acordo com a metodologia de identificação dos ICSCSP utilizada. Aplicando a metodologia canadiana, que inclui apenas doenças crónicas, existiu um decréscimo constante da taxa de ICSCSP do ano 2000 até 2011, de 282,6 para 217,3 int./100 000 hab. (Fig. 4). No entanto, segundo a metodologia espanhola, que inclui também patologias agudas e imunizáveis, houve um crescimento da taxa de internamentos de $2000(1.417,1$ int./100 000 hab.) A 2012 (1.644,5 int./100 000 hab.) (Fig. 4).

Cenários de melhoria: Analisando os cenários de melhoria up the ladder e all the best, ${ }^{2}$ estima-se uma meIhoria possível de acordo com a metodologia canadiana

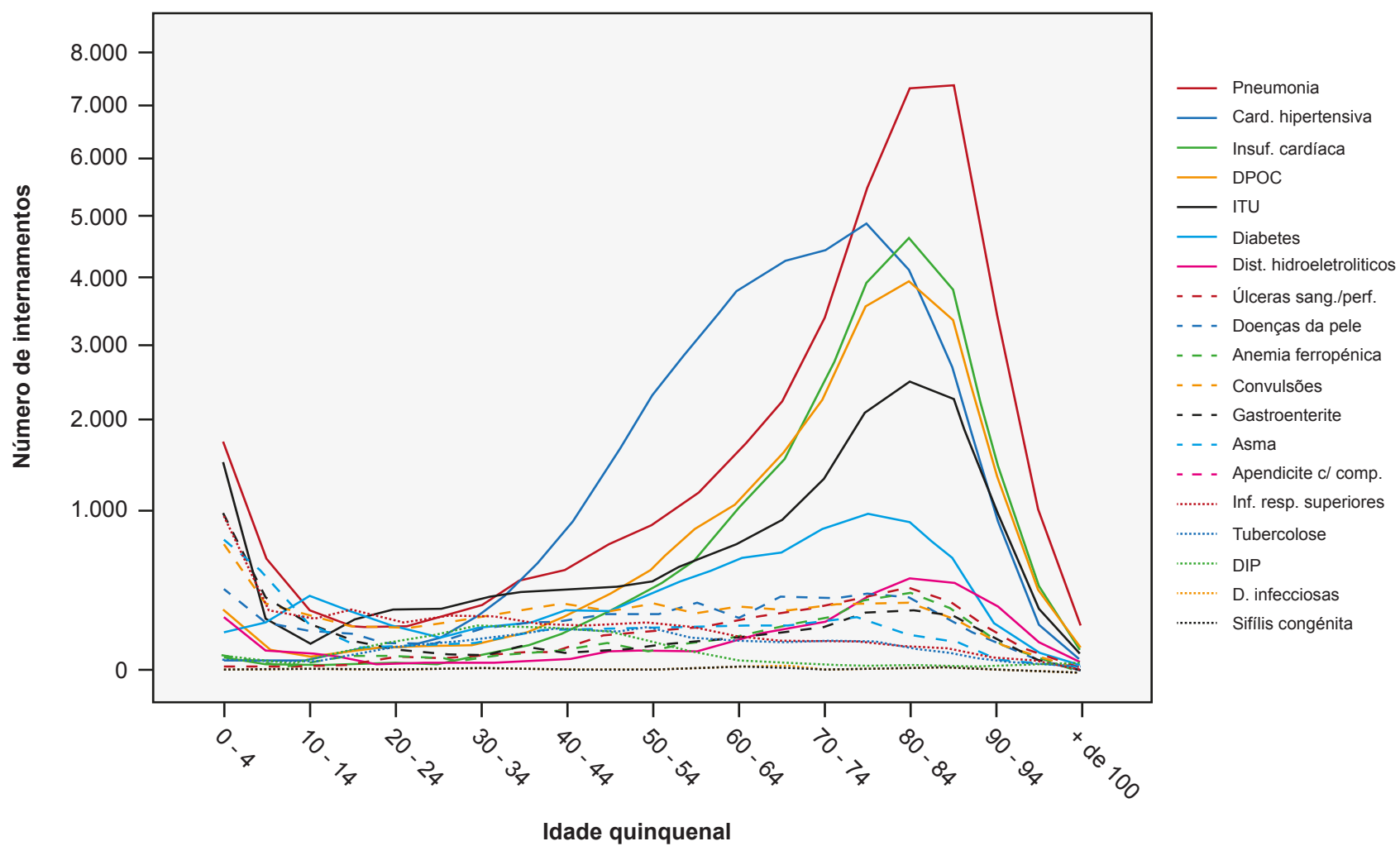

Figura 2 - Distribuição dos ICSCSP por patologia e classe etária (m. espanhola)

DIP: Doença inflamatória pélvica; DPOC: Doença pulmonar obstrutiva crónica; ITU: Infecção do trato urinário 

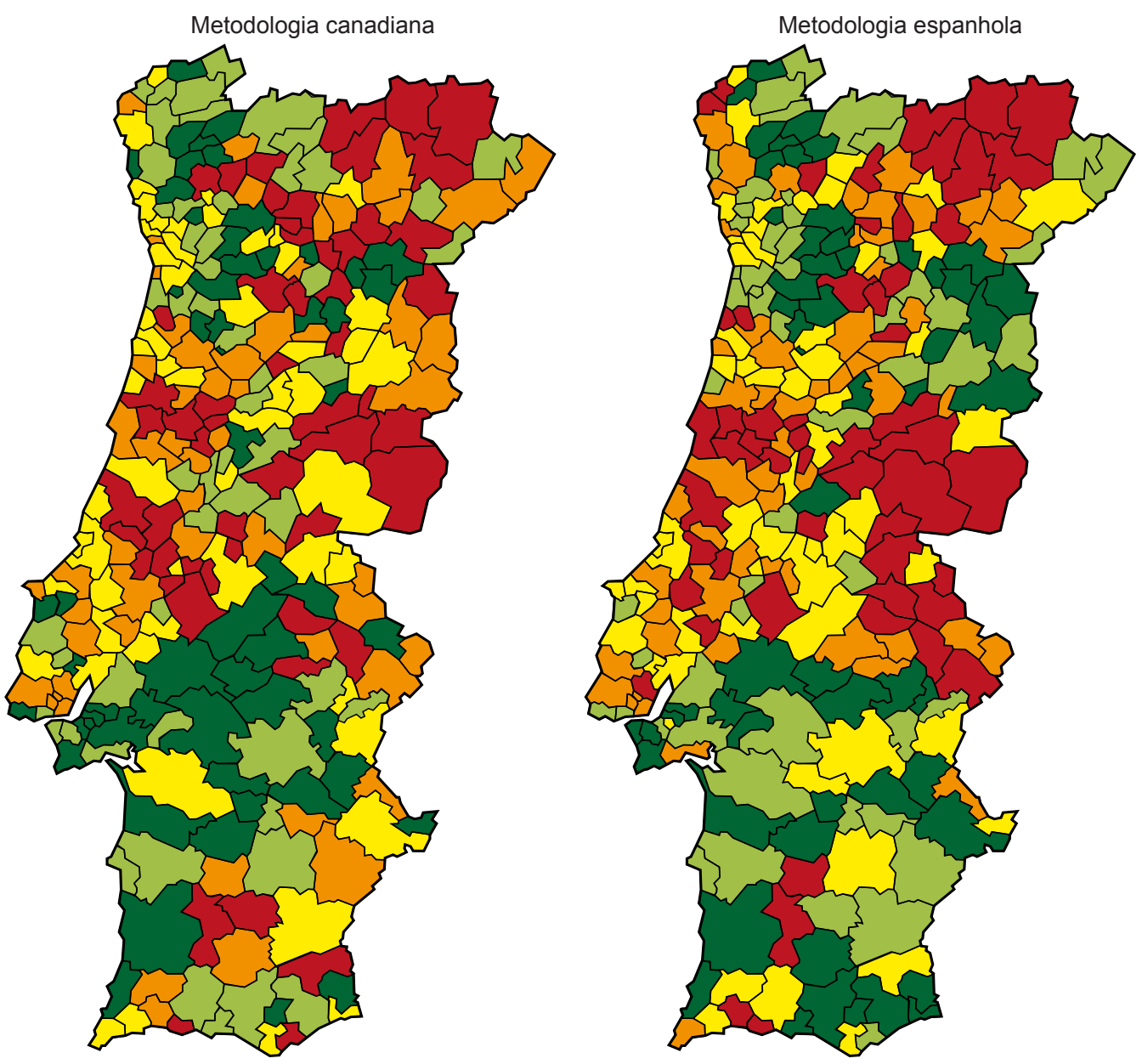

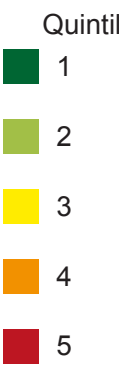

Figura 3 - Distribuição dos ICSCSP no território continental português de acordo com a taxa internamento padronizada $\mathrm{O}$ quintil 1 corresponde às taxas de internamento mais baixas

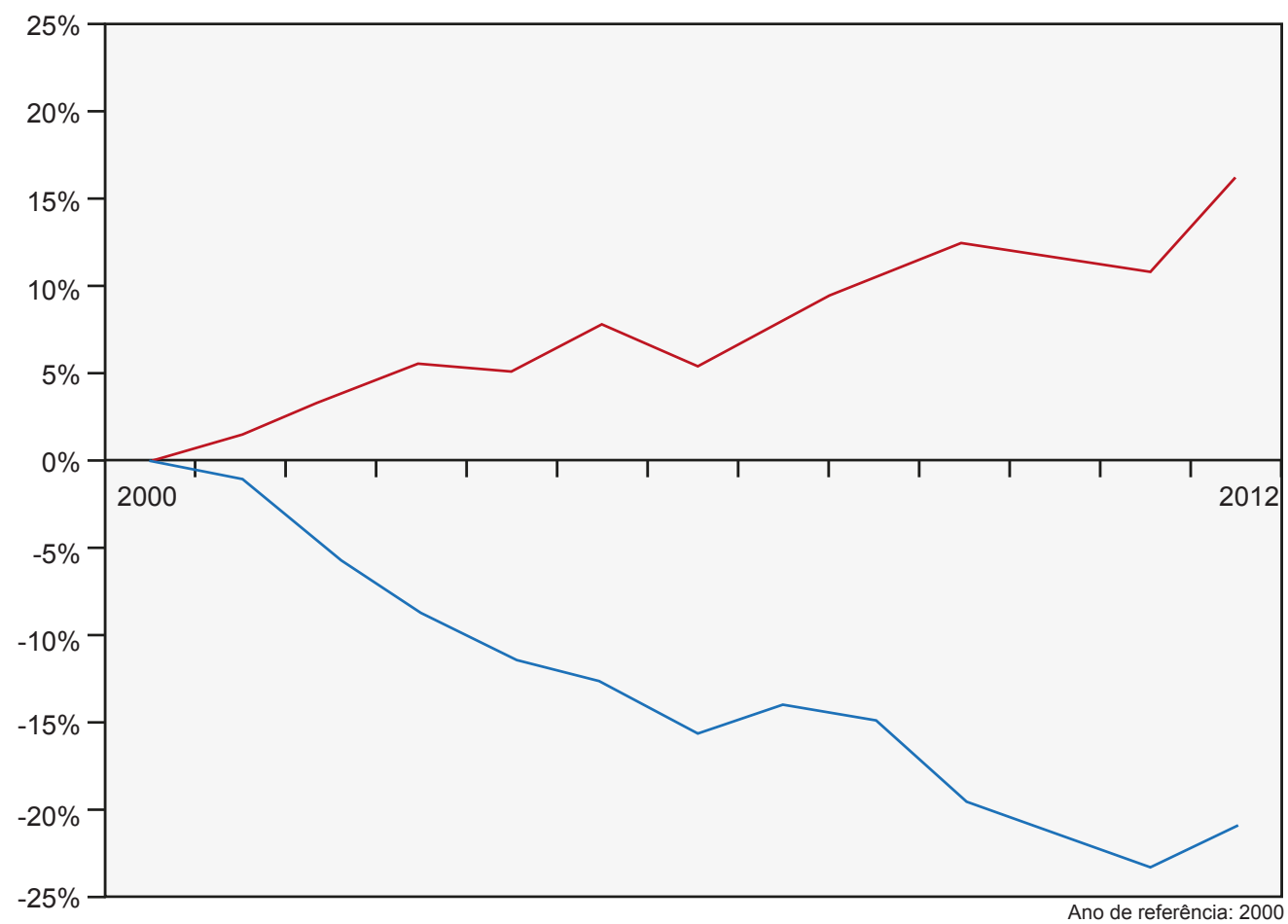

M. espanhola

M. canadiana

Figura 4 - Evolução histórica da variação acumulada (em \%) das taxas de ICSCSP em Portugal de 2000 a 2012 de acordo com duas listas de ICSCSP 
de $20,3 \%$ e $45,9 \%$ respectivamente, e de $43,9 \%$ e $53,5 \%$ segundo a metodologia espanhola. Isto significa uma redução entre 3699 e 86325 internamentos e uma poupança financeira potencial estimada para o Serviço Nacional de Saúde entre 9 M€ e 206,2 M€ (Tabela 2). Na Fig. 5 pode observar-se a dimensão geográfica da melhoria no cenário up the ladder.

Utilização de diferentes metodologias de identificação de ICSCSP: A utilização de diferentes metodologias de identificação dos ICSCSP dá origem a resultados com diferenças consideráveis. Nas Tabelas 3 e 4 podem ser consultados e comparados os principais resultados. Para medir a concordância da classificação em quintis das duas metodologias calculou-se a estatística kappa, sendo o resultado de 0,308 ( $p \leq 0,001)$, correspondendo a uma concordância baixa.

\section{DISCUSSÃO}

Os internamentos evitáveis em Portugal assumem uma dimensão considerável: $4,4 \%$ a $32,5 \%$ dos internamentos por causas médicas. Apesar de em teoria todos os internamentos identificados serem evitáveis, não será possível a eliminação sistemática e completa da ocorrência deste fenómeno. Esta resulta da interacção complexa de uma série de factores que não são todos modificáveis ou controláveis. ${ }^{6,8}$ No entanto o estudo deste fenómeno poderá ter várias potencialidades. A capacidade de avaliação e monitorização de resultados, bem como a identificação de

Tabela 2 - Cenários de melhoria

\begin{tabular}{llllc}
\hline & & & \multicolumn{2}{c}{ Redução } \\
Metodologia & Cenário & N. ${ }^{\circ}$ Int. & $\%$ Int. & Financeira* \\
\hline \multirow{2}{*}{ Canadiana } & Up the ladder & 3699 & $20,3 \%$ & $7558499 €$ \\
& All the best & 8366 & $45,9 \%$ & $17095000 €$ \\
Espanhola & Up the ladder & 69189 & $43,9 \%$ & $189876064 €$ \\
& All the best & 84325 & $53,5 \%$ & $231413940 €$ \\
\hline
\end{tabular}

\footnotetext{
*Estimando o preço médio dos ICSCSP no ano de 2012 de acordo com a Portaria 839-A/2009
}
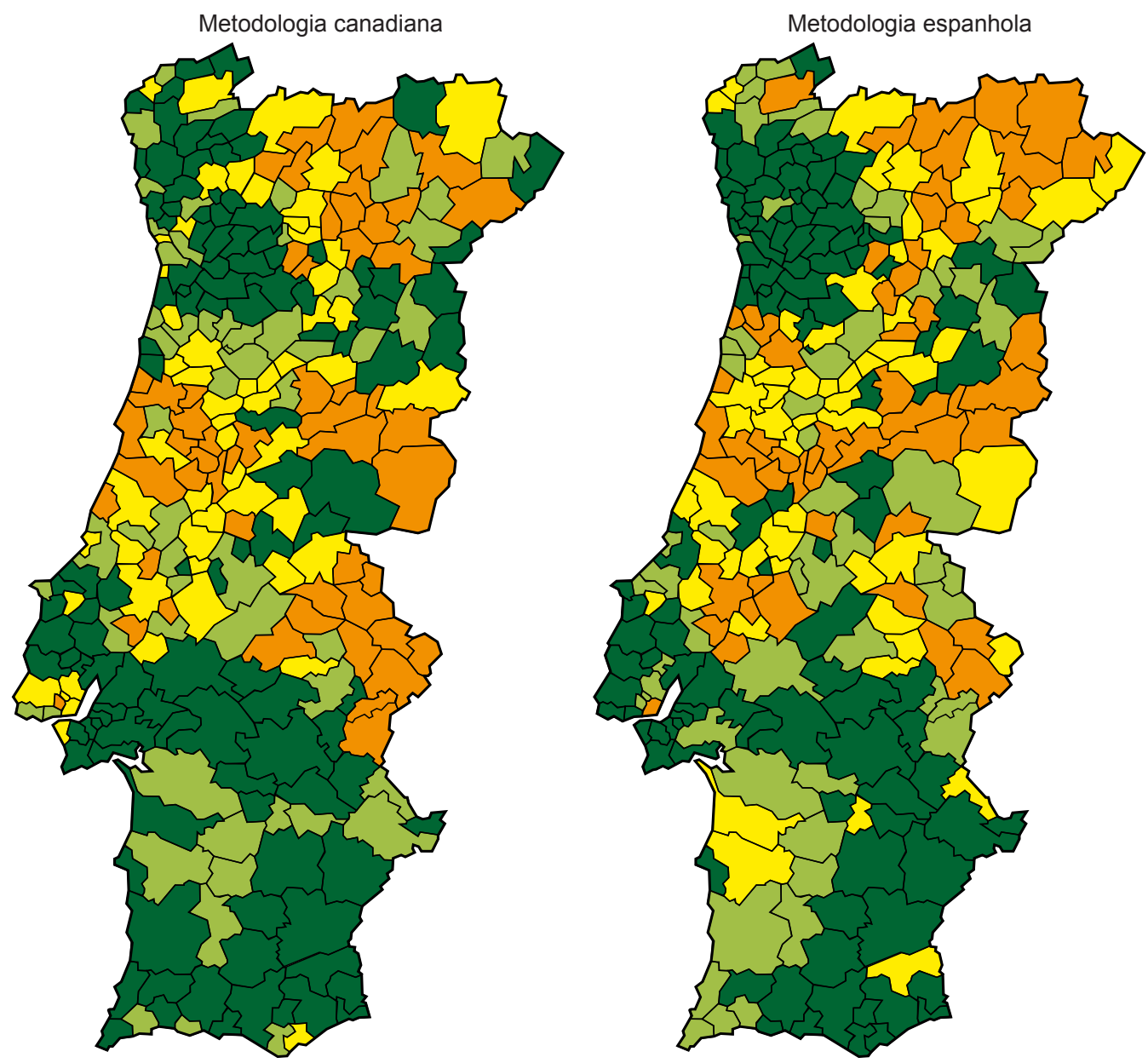

Figura 5 - Distribuição dos ICSCSP no território continental português segundo o cenário de melhoria up the ladder 
Tabela 3 - Caracterização dos ICSCSP segundo diferentes metodologias de identificação

\begin{tabular}{lcc}
\hline & Metodologia canadiana & Metodologia espanhola \\
\hline N. ${ }^{\circ}$ Total de ICSCSP & 22321 & 164061 \\
Taxa de ICSCSP (int./100 000 hab.) & 223,7 & $1.644,5$ \\
$\%$ de internamentos & $2,4 \%$ & $17,7 \%$ \\
$\%$ de int. causas médicas & $4,4 \%$ & $32,5 \%$ \\
$\%$ sexo masculino & $57,0 \%$ & $52,0 \%$ \\
Impacto financeiro* & $45610708 €$ & $450234825 €$ \\
\hline${ }^{*}$ Calculado para o ano de 2012 de acordo com o preço estipulado na Portaria 839-A/2009 &
\end{tabular}

prioridades de intervenção serão os mais importantes.

Como visto anteriormente, é possível identificar o fenómeno com maior especificidade ou sensibilidade (conforme a metodologia utilizada). Esta calibração é importante para o tipo de conclusões que se pretendem tirar. Enquanto uma avaliação mais sensível permite uma melhor avaliação sistémica, uma avaliação mais específica permite comparar realidades distintas com melhor nível de precisão., ${ }^{4,6}$

Também no que respeita à estimativa da representatividade financeira, a metodologia seleccionada para a sua valorização pode influenciar os resultados obtidos: sendo que neste estudo, utilizaram-se os preços de referência das portarias dos Grupos de Diagnóstico Homogéneos. Apesar de se tratar de uma estimativa, outras possibilidades de referência valorativa poderiam ter sido utilizadas, como os preços dos contratos-programa dos hospitais ou os valores de custos dos internamentos em causa. A inespecificidade da primeira opção e a inconsistência actual da contabilidade analítica conduziu à utilização dos preços dos $\mathrm{GDH}$, que também apresentam limitações na sua estruturação, nomeadamente: a falta de conexão com as necessidades em saúde, produção e eficiência hospitalares, ${ }^{33}$ recurso a uma matriz de consumo de recursos americana para o seu cálculo (conhecida por matriz de Maryland) e problemas gerais metodológicos. ${ }^{34}$

A análise dos ICSCSP em Portugal permite uma série de considerações:

1. A distribuição etária da ocorrência dos ICSCSP está de acordo com a literatura internacional, com a ocorrência de dois picos de incidência, um em idade pediátrica e outro nas faixas etárias mais avançadas. .11,14 $^{11}$

2. As patologias mais frequentes nestes picos são distintas. Neste momento, importa frisar a significativa diferença conceptual das metodologias de identificação dos
ICSCSP. Enquanto o método canadiano considera apenas patologias crónicas, o método espanhol acrescenta também as patologias imunizáveis e sensíveis ao diagnóstico e/ou tratamento precoce. Assim sendo, o segundo permite uma avaliação mais abrangente da aç̧ão dos CSP, e consequentemente, identifica um número significativamente superior de ICSCSP. De facto, pelo método espanhol, as principais causas de internamentos evitáveis em ambos os picos etários devem-se a patologia aguda, nomeadamente pneumonia. Seguem-se as patologias crónicas em que o bom controlo é fundamental, havendo concordância entre os métodos na identificação da insuficiência cardíaca e DPOC como patologias responsáveis por grande parte dos internamentos evitáveis.

3. Sendo os ICSCSP um indicador comprovado do acesso e efectividade dos CSP, a ocorrência destes internamentos sugerirá um problema nestas dimensões. Estes factos, embora sirvam de alerta para o planeamento de intervenções de Saúde nacionais, assumem uma importância maior quando analisados em detalhe geográfico. É possível identificar agregados regionais com resultados abaixo e acima da média. O presente estudo tem apenas carácter descritivo, não sendo possível atribuir causalidades, no entanto, é possível identificar regiões em que a intervenção é prioritária. É importante estudar qual a causa destes resultados, sendo decorrentes de eventuais défices de acesso ou de efectividade dos cuidados prestados. Como visto anteriormente, características como a prevalência ou severidade da doença também devem ser tidas em conta para a explicação destes resultados. Será ainda fundamental considerar as características dos prestadores de cuidados, principalmente o acesso aos CSP e a proximidade e acesso aos hospitais. Por último, mas não menos importante, é a influência do nível socioeconómico dos utentes, sabendo

Tabela 4 - Estatísticas descritivas das taxas padronizadas de ICSCSP segundo diferentes metodologias de identificação

\begin{tabular}{lcc}
$\begin{array}{l}\text { Taxa padronizada de ICSCSP por concelho } \\
\text { (int./100 } 000 \text { hab.) }\end{array}$ & Metodologia canadiana & Metodologia espanhola \\
\hline Máximo & 517,8 & 1805,5 \\
Mínimo & 24,1 & 421,0 \\
Média & 185,4 & 999,4 \\
Desvio padrão & 72,6 & 220,8
\end{tabular}


que há uma certa concordância entre as zonas de performance crítica identificadas e níveis socioeconómicos mais baixos.

Quando nos centramos na evolução histórica das taxas de ICSCSP, a sua análise revela uma diferença marcada de tendência conforme a metodologia de identificação dos internamentos. Segundo a metodologia canadiana, houve uma melhoria progressiva desde o ano 2000, atingindo uma redução de cerca de $20 \%$ de ICSCSP em 2012. Segundo a metodologia espanhola houve um agravamento da taxa de ICSCSP em cerca de 16\%. Este facto revela a importância da selecção da metodologia, sendo necessário em Portugal haver consenso sobre o escopo da avaliação deste fenómeno, bem como qual a metodologia mais adequada para o fazer. Esta diferença releva também a importância de avaliar as várias dimensões de intervenção dos CSP. Enquanto parece existir uma melhoria na gestão da doença crónica, parece haver simultaneamente uma falta de resposta no diagnóstico e/ou tratamento da doença aguda. Sendo este fenómeno complexo, uma possível explicação será o envelhecimento da população com o conhecido aumento da morbilidade, originando um aumento das necessidades de cuidados eventualmente não acompanhada pela sua oferta.

A construção de cenários de melhorias alicerçados na realidade descrita torna-se um exercício importante. Os dois cenários propostos reflectem duas possibilidades distintas. Enquanto o cenário up the ladder assume-se como mais próximo de um eventual movimento de melhoria, o cenário all the best, mais utópico, demonstra o maior potencial concretizável. Assim, os cálculos efectuados apontam para melhorias significativas, mesmo no cenário mais conservador (redução de 3699 int./ano). Estes resultados são relevantes sobretudo ao nível da qualidade percepcionada pelos utentes, que entendem um internamento como um evento disruptivo e adverso. Além disso, estes ICSCSP podem também associar-se a infecções nosocomiais e/ou a procedimentos desnecessários. ${ }^{5} \mathrm{O}$ impacto financeiro para o SNS é difícil de estimar, uma vez que não há forma de determinação do custo efectivo de cada internamento. A utilização do preço médio dos ICSCSP de acordo com a tabela de preços definida pela ACSS assume-se como o proxy possível. Uma poupança potencial estimada entre 7,5 M€ e 231,4 M€, não representando muito mais de 2,5\% do total das despesas do estado com a Saúde, será um contributo importante para a redução do desperdício no SNS.

\section{CONCLUSÃO}

A principal conclusão é que a utilização de diferentes metodologias de identificação dos ICSCSP tem influência nos resultados descritos. Os ICSCSP são em Portugal, de facto, um fenómeno relevante e importante que, por reflectirem uma falta de acesso ou inefectividade dos CSP, podem traduzir potenciais de melhoria.

Fixando a observação em cada uma das metodologias, é possível identificar prioridades de intervenção em termos de faixas etárias, tipo de patologias e regiões geográficas. Os ICSCSP são mais frequentes nas idades pediátricas e a partir da quinta década de vida. As doenças agudas que mais frequentemente resultam em internamentos evitáveis são pneumonia e pielonefrites. A insuficiência cardíaca, DPOC e cardiopatia hipertensiva são as principais doenças crónicas responsáveis por ICSCSP. Existem aglomerados regionais de resultados acima e abaixo da média nacional. Apesar de parecer haver uma diminuição dos ICSCSP devidos a doença crónica (segundo a metodologia canadiana), parece haver um aumento quando considerados também os internamentos por doenças agudas e imunizáveis (metodologia espanhola).

Estimam-se cenários de melhoria entre 20,3\% e 53,5\% de redução de ICSCSP, traduzindo uma poupança financeira potencial estimada para o SNS entre 7,5 M€ e 231,4 M€.

Para uma caracterização mais clara e adequada deste fenómeno em Portugal é necessário consensualizar a meIhor metodologia de identificação dos ICSCSP. Este será o próximo passo dos autores nesta linha de investigação. Seguir-se-ão, ainda, iniciativas com o objectivo de obter um impacto real na redução dos internamentos evitáveis.

\section{AGRADECIMENTOS}

Os autores agradecem à ACSS a disponibilização dos dados e a Isabel Andrade a colaboração especialmente dificultada pelos constrangimentos da distância e dificuldade de acesso à internet.

\section{PROTECÇÃO DE PESSOAS E ANIMAIS}

Os autores declaram que os procedimentos seguidos estavam de acordo com os regulamentos estabelecidos pelos responsáveis da Comissão de Investigação Clínica e Ética e de acordo com a Declaração de Helsínquia da Associação Médica Mundial.

\section{CONFIDENCIALIDADE DOS DADOS}

Os autores declaram ter seguido os protocolos do seu centro de trabalho acerca da publicação de dados.

\section{CONFLITOS DE INTERESSE}

Os autores não têm conflitos de interesse a declarar.

\section{FONTES DE FINANCIAMENTO}

Os autores não receberam qualquer tipo de financiamento para a realização desde estudo.

\section{REFERÊNCIAS}

1. Sanchez M, Vellanky S, Herring J, Liang J, Jia H. Variations in Canadian rates of hospitalization for ambulatory care sensitive conditions. Healthc Q. 2008;11:20-2.

2. Tian Y, Dixon A, Gao H. Data briefing: emergency hospital admissions for ambulatory care-sensitive conditions: identifying the potential for reductions. London: The King's Fund; 2012.

3. Ansari Z, Barbetti T, Carson NJ, Auckland MJ, Cicuttini F. The Victorian ambulatory care sensitive conditions study: rural and urban perspectives. Soz Praventivmed. 2003;48:33-43.

4. Homar JC, Matutano CC. La evaluación de la atención primaria y las 
hospitalizaciones por ambulatory care sensitive conditions. Marco conceptual. Aten Primaria. 2003;31:61-5

5. Homar J, Espinoza M, Ruiz E. Hospitalizations preventable by timely and effective primary health care. Aten Primaria. 2003;31:6-17.

6. Nedel F, Facchini L, Bastos J, Martín-Mateo M. Conceptual and methodological aspects in the study of hospitalizations for ambulatory care sensitive conditions. Cien Saude Colet. 2011;16:1145-54.

7. Rutstein DD, Berenberg W, Chalmers TC, Child CG, Fishman AP, Perrin EB. Measuring the quality of medical care: a clinical method. N Engl $J$ Med. 1976;294:582-8.

8. Caminal J, Starfield B, Sánchez C, Casanova C, Morales M. The role of primary care in preventing ambulatory care sensitive conditions. Eur J Public Health. 2004;14:246-51.

9. Brown A, Goldacre M, Hicks N, Rourke J, McMurtry R, Brown J, et al. Hospitalization for ambulatory care-sensitive conditions: a method for comparative access and quality studies using routinely collected statistics. Can J Public Health. 2001;92:155-9.

10. Alfradique ME, Bonolo PF, Dourado I, Lima-Costa MF, Macinko J, Mendonça CS, et al. Ambulatory care sensitive hospitalizations: elaboration of Brazilian list as a tool for measuring health system performance (Project ICSAP--Brazil). Cad Saude Publica. 2009;25:1337-49.

11. Caminal J, Mundet X, Ponsà J, Sánchez E, Casanova C. Las hospitalizaciones por ambulatory care sensitive conditions: selección del listado de códigos de diagnóstico válidos para España. Gac Sanit. 2001;15:128-41.

12. Solberg LI, Peterson KE, Ellis RW, Romness K, Rohrenbach E, Thell T, et al. The Minnesota project: a focused approach to ambulatory quality assessment. Inquiry. 1990;27:359-67.

13. Weissman J, Gatsonis C, Epstein A. Rates of avoidable hospitalization by insurance status in Massachusetts and Maryland. JAMA;1992;268:2388-94.

14. Rizza P, Bianco A, Pavia M, Angelillo IF. Preventable hospitalization and access to primary health care in an area of Southern Italy. BMC Health Serv Res. 2007;7:134.

15. Hossain MM, Laditka JN. Using hospitalization for ambulatory care sensitive conditions to measure access to primary health care: an application of spatial structural equation modeling. Int J Health Geogr. 2009;8:51.

16. Menec VH, Sirski M, Attawar D, Katz A. Does continuity of care with a family physician reduce hospitalizations among older adults? J Health Serv Res Policy. 2006;11:196-201.

17. Roos LL, Walld R, Uhanova J, Bond R. Physician visits, hospitalizations, and socioeconomic status: ambulatory care sensitive conditions in a canadian setting. Health Serv Res. 2005;40:1167-85.

18. Pirani M, Schifano P, Agabiti N, Davoli M, Caranci N, Perucci CA Potentially avoidable hospitalisation in Bologna, 1997-2000: temporal trend and differences by income level. Epidemiol Prev. 2006;30:169-77.

19. Agabiti N, Pirani M, Schifano P, Cesaroni G, Davoli M, Bisanti L, et al. Income level and chronic ambulatory care sensitive conditions in adults: a multicity population-based study in Italy. BMC Public Health. 2009;9:457
20. Magan P, Otero A, Alberquilla A, Ribera JM. Geographic variations in avoidable hospitalizations in the elderly, in a health system with universal coverage. BMC Health Serv Res. 2008;8:42.

21. Bermúdez-Tamayo $C$, Márquez-Calderón $S$, Rodríguez del Aguila MM, Perea-Milla López E, Ortiz Espinosa J. Organizational characteristics of primary care and hospitalization for to the main ambulatory care sensitive conditions. Aten primaria. 2004;33:305-11.

22. Millman M. Access to health care in America. $1^{\text {st }}$ ed. Washington: National Academy Press; 1993.

23. Billings J, Zeitel L, Lukomnik J, Carey TS, Blank AE, Newman L. Impact of socioeconomic status on hospital use in New York City. Health Aff. 1993;12:162-73.

24. Sanderson C, Dixon J. Conditions for which onset or hospital admission is potentially preventable by timely and effective ambulatory care. $\mathrm{J} H$ Heal Serv Res Policy. 2000;5:222-30.

25. AHRQ. AHRQ Quality Indicators: quide to prevention quality indicators: hospital admission for Ambulatory Care Sensitive Conditions. 4th rev ed Rockville: Agency for Healthcare Research and Quality; 2004.

26. Canadian Institute for Health Information. Technical Note: Ambulatory Care Sensitive Conditions (ACSC) 2008. Ottawa (ON): CIHI; 2008. [consultado 2015 Jan 20]. Disponível em: http://www.cihi.ca/cihi-extportal/internet/en/document/health+system+performance/indicators/ health/tech_acsc 2011

27. Ministério da Saúde. Gabinete Técnico do PNS 2011/2016. Cálculo de internamentos evitáveis por prevenção primária e internamentos por causas sensíveis a cuidados de ambulatório. 2010. Gabinete Técnico do PNS 2011/2016. [consultado 2015 Jan 20]. Disponível em: http://www. uc.pt/fluc/gigs/GeoHealthS/doc_apoio/Contexto-de-InternamentosEvitaveis.pdf

28. Carneiro C. Essays on health economics: equity and access to healthcare and public hospital performance under corporatized management. Porto: Faculdade de Economia. Universidade do Porto; 2011. Tese. [consultado 2015 Jan 18]. Disponível em: http://repositorioaberto.up.pt/handle/10216/56320?mode=full.

29. Sais C, Lopes H, Completo J, Delgado M, Casas M. Ambulatory care sensitive conditions: impacte do internamento dos doentes crónicos no SNS. Lisboa: IASIST Portugal; 2013.

30. Ahmad O, Boschi-Pinto C, Lopez A, Murray C, Lozano R, Inoue M Age standardization of rates: a new WHO standard. World Health Organization: Copenhagen; 2001.

31. Cohen J. A coefficient of agreement for nominal scales. Educ Psycho Meas. 1960;20:37-46.

32. Sarmento J. Internamentos por causas sensíveis a cuidados de ambulatório em contexto de integração vertical. Lisboa: Escola Nacional de Saúde Pública. Universidade Nova de Lisboa; 2013. Tese. [consultado 2015 Jan 20]. Disponível em: http://run.unl.pt/handle/10362/9631.

33. Santana R. O financiamento hospitalar e a definição de preços. Rev Saude Publica. 2005;5:93-118

34. Tribunal de Contas. Auditoria ao sistema de pagamentos e de formação de preços pagos às unidades hospitalares do Serviço Nacional de Saúde (Relatório n. ${ }^{\circ}$ 30/2011 $2^{a}$ Secção - Processso n. ${ }^{\circ} 42 / 2010$ Audit). Lisboa: Tribunal de Contas; 2011. 
João SARMENTO, Conceição ALVES, Paula OLIVEIRA, Rita SEBASTIÃO, Rui SANTANA

\section{Caracterização e Evolução dos Internamentos Evitáveis em Portugal: Impacto de Duas Abordagens Metodológicas}

Acta Med Port 2015:28:590-600

Publicado pela Acta Médica Portuguesa, a Revista Científica da Ordem dos Médicos

Av. Almirante Gago Coutinho, 151

1749-084 Lisboa, Portugal.

Tel: +351218428 215

E-mail: submissao@actamedicaportuguesa.com

www.actamedicaportuguesa.com

ISSN:0870-399X | e-ISSN: 1646-0758

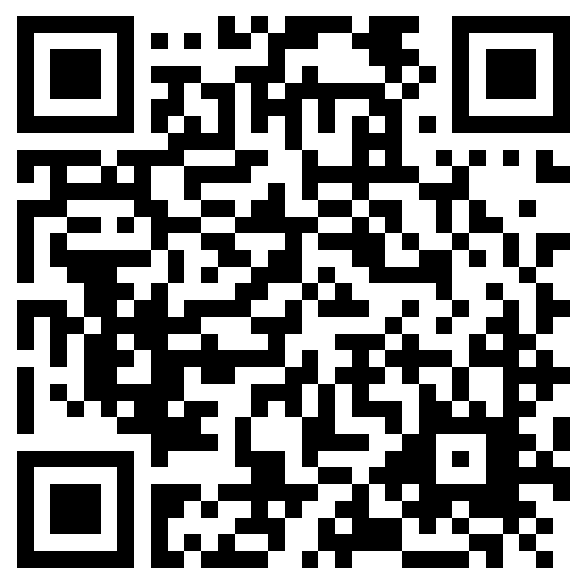

